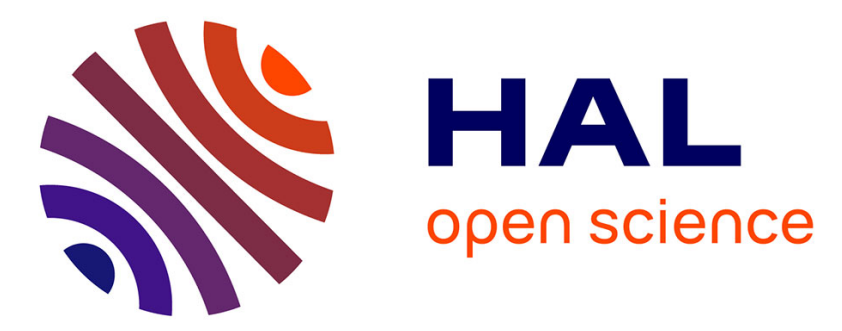

\title{
Comments on the mixture detection rule used in SPC control charts
}

Benjamin Fournier, Nicolas Rupin, Maxence Bigerelle, Denis Najjar, Alain Iost

\section{To cite this version:}

Benjamin Fournier, Nicolas Rupin, Maxence Bigerelle, Denis Najjar, Alain Iost. Comments on the mixture detection rule used in SPC control charts. Communications in Statistics - Simulation and Computation, 2007, 36 (6), pp.1321-1331. 10.1080/03610910701569630 . hal-01325904

\section{HAL Id: hal-01325904 https://hal.science/hal-01325904}

Submitted on 22 Nov 2017

HAL is a multi-disciplinary open access archive for the deposit and dissemination of scientific research documents, whether they are published or not. The documents may come from teaching and research institutions in France or abroad, or from public or private research centers.
L'archive ouverte pluridisciplinaire HAL, est destinée au dépôt et à la diffusion de documents scientifiques de niveau recherche, publiés ou non, émanant des établissements d'enseignement et de recherche français ou étrangers, des laboratoires publics ou privés. 


\title{
Comments on the Mixture Detection Rule Used in SPC Control Charts
}

\author{
B. FOURNIER ${ }^{1}$, N. RUPIN ${ }^{1}$, M. BIGERELLE ${ }^{1,2}$, \\ D. NAJJAR ${ }^{1}$, AND A. IOST $^{1}$
}

${ }^{1}$ Equipe Caractérisation et Propriétés des Périsurfaces, Lille, France

${ }^{2}$ Laboratoire Roberval, Centre de Recherches de Royallieu,

Compiègne, France

\begin{abstract}
When calculating independently the false alarm rate of the eight usual runs rules used in SPC control chart, it appears that the proposed rule designed to detect mixture patterns corresponds to a Type-I error strongly lower than the seven other rules. This discrepancy is underlined and the mixture rule is showed to be useless both for in-control and out-of-control processes. Thus a modification of the mixture detection rule is proposed and the impact of this new mixture rule is then illustrated and discussed using Monte Carlo calculations.
\end{abstract}

Keywords Average run length; Mixture; Statistical process control; Test for special causes; Type-I error; Western electric rules.

Mathematics Subject Classification $62-09 ; 62 \mathrm{C} 99 ; 62 \mathrm{~F} 03 ; 68-04 ; 68 \mathrm{P} 99$.

\section{Introduction}

Statistical process control (SPC) control charts have been used in industry since the 1920's. After the introduction of the first and most widely known Shewhart chart (Shewhart, 1931), additional runs rules have been proposed to detect special causes. The eight most widespread runs rules were expressed in the Western Electric Handbook (Western Electric Company, 1956) and later discussed by Nelson (1984, 1985):

Rule 1: One point beyond Zone A.

Rule 2: Nine points in a row in Zone $\mathrm{C}$ or beyond.

Rule 3: Six points in a row steadily increasing or decreasing.

Rule 4: Fourteen points in a row alternating up and down.

Rule 5: Two out of three points in a row in Zone A or beyond.

Received August 18, 2006; Accepted April 12, 2007

Address correspondence to B. Fournier, CEA Saclay; DEN/DANS/DMN/SRMA, 91191 Gif-surYvette Cedex, France; E-mail: benjamin.fournier@cea.fr 
Rule 6: Four out of five points in a row in Zone B or beyond.

Rule 7: Fifteen points in a row in Zone $\mathrm{C}$ (above and below centreline)

Rule 8: Eight points in a row on both sides of centreline with none in Zone C.

where Zone $\mathrm{C}$ is the area between $\hat{\mu}-\hat{\sigma}$ and $\hat{\mu}+\hat{\sigma}$; Zone $\mathrm{B}$ is the area between $\hat{\mu}-2 \hat{\sigma}$ and $\hat{\mu}-\hat{\sigma}$ and between $\hat{\mu}+\hat{\sigma}$ and $\hat{\mu}+2 \hat{\sigma}$; Zone $\mathrm{A}$ is the area between $\hat{\mu}-3 \hat{\sigma}$ and $\hat{\mu}-2 \hat{\sigma}$ and between $\hat{\mu}+2 \hat{\sigma}$ and $\hat{\mu}+3 \hat{\sigma}$, and $\hat{\mu}$ and $\hat{\sigma}$ being, respectively, the estimated mean and standard deviation of the process data.

The present article aims at underlying the fact that all these rules are not equivalent and, more specifically, that Rule 8 does not correspond to the same false alarm rate (FAR: the test falsely signals the presence of a special cause). This specific point must be highlighted, so that SPC practitioners can correctly interpret the data resulting from the use of these very common rules. In order to reduce this discrepancy between these rules, a modification of the mixture rule (Rule 8 ) is then proposed and the influence on the FAR for usual combinations of rules is discussed.

\section{Individual False Alarm Rates}

The patterns detected by these rules were designed to lead to a FAR roughly equal to the corresponding probability of Rule 1 (which is close to $0.27 \%$ ) as quoted by several authors (Charnes, 1995; Nelson, 1985). Nevertheless, a first difference in the rules definition exists between practitioners. Indeed, as far as Rule 2 is concerned, several authors consider the detection of nine points (Charnes, 1995; Nelson, 1984, 1985), whereas others consider a series of eight points (Champ and Woodall, 1987; Walter et al., 1991; Western Electric Company, 1956; Zhang and $\mathrm{Wu}, 2005)$. In the first case a pattern with 9 following points on the same side of the centreline (in the following this definition of Rule 2 will be named the Nelson definition) approximately corresponds to a FAR of $0.195 \%$, whereas for 8 points (in the following this definition of Rule 2 will then be named the Western definition) the FAR is close to $0.391 \%$.

Besides, a serious discrepancy exists between rule number eight (which is aimed at detecting the presence of an undesired mixture between two distinct populations) and the seven others. Indeed the FAR of rule $8\left(\approx(1-0.683)^{8}\right)$ is approximately thirty times lower than that of the other seven rules (around $0.27 \%$ ) (see Table 1). Table 1 also presents the ARL values for the eight individual laws (each simulation tests only one of the eight rules). These values are calculated either by analytical calculations or using Monte Carlo simulations. They are shown as an example since, in practical cases, there is no denying that the number and size of subgroups of data must be taken into account (Adams et al., 2001; Walter et al., 1991), which has not been done here.

Table 1

FAR for individual rules and their ARL (the value into brackets corresponds to the Western definition of Rule 2 that considers only 8 following points)

\begin{tabular}{lcccccccc}
\hline Rule $n^{\circ}$ & 1 & 2 & 3 & 4 & 5 & 6 & 7 & 8 \\
\hline FAR (\%) & 0.27 & $0.19(0.39)$ & 0.28 & 0.29 & 0.31 & 0.45 & 0.33 & 0.01 \\
ARL & 370.2 & $510.8(256.4)$ & 422.0 & 601.2 & 510.2 & 291.1 & 964.5 & 14084 \\
\hline
\end{tabular}


Table 2

ARL values for three combinations of rules (the values into brackets correspond to the Western definition of Rule 2 that considers only eight following points)

\begin{tabular}{lccc}
\hline Combination & $\mathrm{C}_{123456}$ & $\mathrm{C}_{1234567}$ & $\mathrm{C}_{12345678}$ \\
\hline ARL & $79.0(70.0)$ & $73.5(65.8)$ & $73.5(65.8)$ \\
\hline
\end{tabular}

Such a very low FAR of Rule 8 has already been reported in Walter (1991). Even though this test is often advocated to be used additionally to the others, this difference must be borne in mind if it is applied to detect the presence of a mixture.

\section{In-control Process: Power of Rule 8}

The previous results were obtained for all the rules applied individually. However, since a multiple testing procedure is carried out when applying a combination of several rules, it increases the power of the control chart and, as a consequence, the global FAR (Champ and Woodall, 1987). The only way to obtain the global FAR for a complex combination of rules is then to calculate their ARL by way of computer simulations ${ }^{1}$, since there is no independence between the rules (Champ, 1992; Zhang and $\mathrm{Wu}, 2005)$.

In reference works, the SPC user is advised to use Rule 8 in combination with the seven others, as a complementary test. Table 2 presents the ARL of control charts combining the six, seven, and eight rules together (that are noted $\mathrm{C}_{123456}, \mathrm{C}_{1234567}, \mathrm{C}_{12345678}$, according to Champ and Woodall, 1987). These results are calculated on the mean of $10^{6}$ runs of randomly generated Gaussian data. It can be noticed that adding Rule 7 to the 6 previous ones significantly reduces the ARL of the control charts, whereas the use of Rule 8 does not reduce the ARL anymore. It means that Rule 8 does not improve the power of the control chart when it is used as commonly advocated. Table 3 shows that the addition of Rule 8 does not significantly modify the ARL of each rule when $\mathrm{C}_{1234567}$ and $\mathrm{C}_{12345678}$ are compared.

Moreover, when tested in combination with the seven other rules, the pattern of the mixture rule is almost never detected (less frequently than $1 \%$ ), as can be seen in Table 4, where the percentage of detection of each abnormal pattern is given.

These results show that, when Rule 8 is applied as usually recommended, it is simply useless since it is almost never detected in the case of in-control data.

If Rule 8 is used in conjunction with only one of two other rules, it may increase more significantly the sensitivity of the control chart. That is the reason why the 255 possible combinations of rules where tested. For a given combination, the addition of Rule 8 led to a decrease in the ARL $\left(\frac{A R L^{\text {without Rule } 8}-A R L^{\text {with Rule } 8}}{A R L^{\text {wihhout Rule } 8}} \times 100\right)$ smaller than $5 \%$ in all cases (in $75 \%$ of the possible combinations, the ARL decrease is even smaller than 1\%). Moreover, in all the possible 128 control charts involving

${ }^{1}$ At each computation step one value is randomly generated from a standard Gaussian distribution. All the selected rules in the chosen combination are then tested. The algorithm stops as soon as one of these rules is infringed. This simulation is repeated a large number of times $\left(10^{6}\right.$ times in the present case) and the global ARL is the average of the total number of data generated for all the simulations. 
Table 3

Influence of Rule 8 on the ARL of each rule for two different combinations of them in the case of the Nelson definition of Rule 2 (values into brackets correspond to the Western definition of Rule 2)

\begin{tabular}{lcccccccc}
\hline & $\begin{array}{c}\text { ARL of } \\
\text { Combination }\end{array}$ & $\begin{array}{c}\text { ARL of } \\
\text { Rule 1 }\end{array}$ & $\begin{array}{c}\text { ARL of } 2 \\
\text { Rule 3 }\end{array}$ & $\begin{array}{c}\text { ARL of } \\
\text { Rule 4 }\end{array}$ & $\begin{array}{c}\text { ARL of } \\
\text { Rule 5 }\end{array}$ & $\begin{array}{c}\text { ARL of } \\
\text { Rule 6 }\end{array}$ & $\begin{array}{c}\text { ARL of } \\
\text { Rule 7 }\end{array}$ & $\begin{array}{c}\text { ARL of } \\
\text { Rule 8 }\end{array}$ \\
\hline $\mathrm{C}_{1234567}$ & 68.8 & 76.0 & 73.4 & 79.9 & 70.5 & 72.4 & 81.4 & \\
& $(61)$ & $(67.2)$ & $(66)$ & $(72.4)$ & $(62.5)$ & $(64.9)$ & $(73)$ & \\
$\mathrm{C}_{12345678}$ & 69.0 & 75.8 & 73.5 & 80.0 & 70.3 & 72.5 & 80.5 & 75.7 \\
& $(61.4)$ & $(67.2)$ & $(65.9)$ & $(72.7)$ & $(62.4)$ & $(64.8)$ & $(73.3)$ & $(67.2)$ \\
\hline
\end{tabular}

Rule 8 , a false alarm is obtained thanks to Rule 8 in less than $5 \%$ of the samples. Therefore, whichever the rules in combination of which the mixture rule is used, it turns out that, when data is simulated from a standard Gaussian distribution (in-control conditions), it is virtually useless.

\section{Mixed Process: Power of Rule 8}

Under in-control conditions, the pattern of Rule 8 is seldom detected as the other rules react before. Nevertheless, Rule 8 is designed to detect a mixture (Western Electric Company, 1956), it is thus possible that, when the data is actually coming from two distinct distributions, Rule 8 should be the first to detect it. In order to test the power of Rule 8 under an out-of-control mixed process, two simple types of mixtures were simulated: a symmetrical one [Fig. 1(a)] and a weighted one which is more general [Fig. 1(b)]. It must be noticed that the present illustrative simulations limited by the control chart limits were built in a first stage, when the process was in-control. It is assumed that all relevant tests for multimodality or mixtures (Efron and Tibshirani, 1993; Minnotte, 1992; Mueller and Sawitzki, 1991) were performed and that during this first stage no mixture existed.

\subsection{Symmetrical Mixture}

The simplest case of mixture consists in considering data coming from two standard Gaussian distributions shifted in a symmetrical way compared to the mean value 0 .

Table 4

Influence of Rule 8 on the percentage of false alarms due to each rule for two control charts for the Nelson definition of Rule 2 (values into brackets correspond to the Western definition)

\begin{tabular}{lcccccccc}
\hline Combination & Rule 1 & Rule 2 & Rule 3 & Rule 4 & Rule 5 & Rule 6 & Rule 7 & Rule 8 \\
\hline 1234567 & 19.5 & 11.9 & 15.9 & 10.7 & 11.1 & 22.0 & 8.9 & \\
& $(17.5)$ & $(22)$ & $(13.9)$ & $(9.3)$ & $(11.7)$ & $(19.4)$ & $(6.2)$ & \\
12345678 & 19.4 & 11.9 & 15.9 & 10.6 & 13.0 & 22.1 & 6.9 & 0.17 \\
& $(17.4)$ & $(22)$ & $(14)$ & $(9.3)$ & $(11.6)$ & $(19.5)$ & $(6.1)$ & $(0.16)$ \\
\hline
\end{tabular}




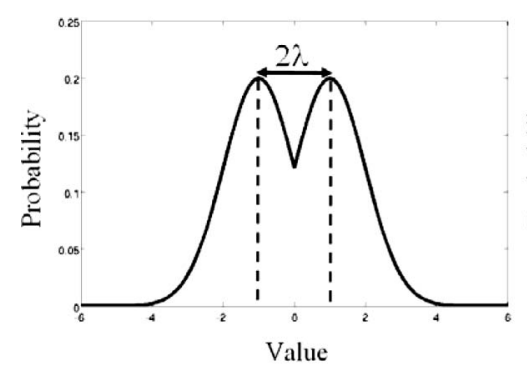

(a)

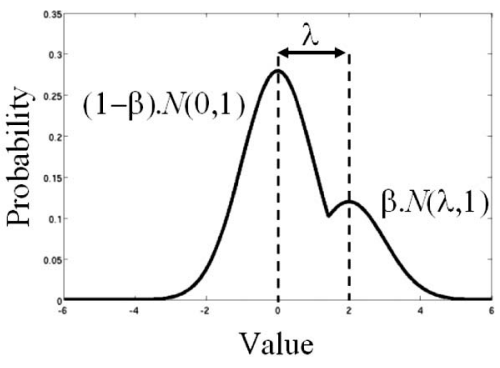

(b)

Figure 1. (a) Symmetrical mixture of two standard Gaussian distributions shifted of a value $\lambda$ (in this case $\lambda=1$ ); (b) non-symmetrical mixture distribution (in this case $\beta=0.3$ and $\lambda=2$ ).

This symmetric process, is characterized by a constant null mean and a standard deviation increasing with the $\lambda$ shift.

The eight rules (with Rule 2 defined as in Nelson, 1984, 1985) were tested together on data coming from such mixtures for values of $\lambda$ varying from $0-2$. As expected, Fig. 2 shows that the global ARL decreases as the mixture shift increases: the mixture is detected more and more quickly. Nevertheless, we can notice in Fig. 3 that the detection of the mixture pattern is not due to Rule 8: indeed, even though Rule 8 is more frequently detected when the data comes from two shifted distributions, it is never detected in more than $2 \%$ of all the data samples. The detection of the mixture is mainly due to Rules 1 and 5.

\subsection{Non Symmetrical Mixture}

Another simple case of mixture can be simulated by randomly generating data coming from two distinct standard Gaussian distributions, with only one of the two

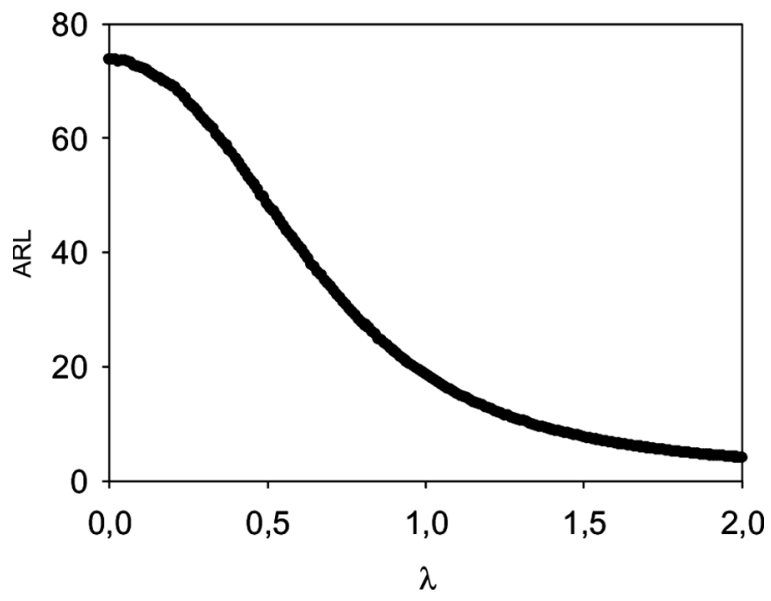

Figure 2. Evolution of the global ARL obtained for the combination of the eight rules as a function of the shift of the symmetrical mixture distribution 


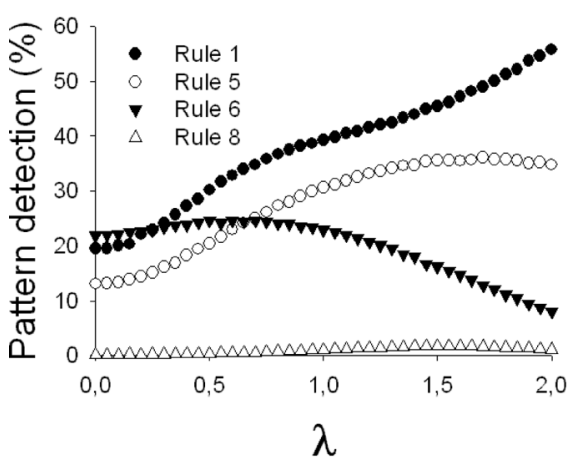

(a)

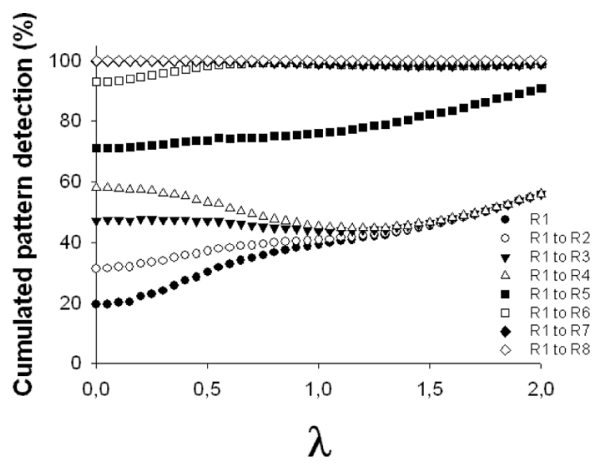

(b)

Figure 3. Percentage of detection of the mixture due to (a) the three most frequently detected patterns (Rules 1, 5,6) and Rule 8. The cumulated percentages of detection shown in (b) highlight the fact that Rules 2, 4, 7, and 8 play a minor role in the detection of a symmetrical mixture.

being shifted from the initial null mean of a value $\lambda$. Additionally, only a fraction $\beta$ of the data comes from the shifted distribution.

The evolution of the global ARL obtained using the same eight rules together is plotted in Fig. 4 for $\lambda$ varying from $0-2$ and $\beta$ varying from $0-0.5$ (for higher values of $\beta$, the observed tendencies do not change anymore). When these two parameters increase the ARL decreases, which means that, as expected, the more mixed the data is, the faster it is detected. The sharpest decrease in the ARL is found for $\lambda=2$ and increases with the values of $\beta$.

Nevertheless, here again, the detection of the mixture is actually not due to Rule 8 , as can be noticed in Fig. 5. Figure 5(b) shows that Rule 8 is very seldom infringed whatever the values of $\lambda$ and $\beta$ (it is always detected in less than $0.2 \%$ of the cases), whereas the most frequently detected pattern corresponds to Rule 1 [Fig. 5(a)].

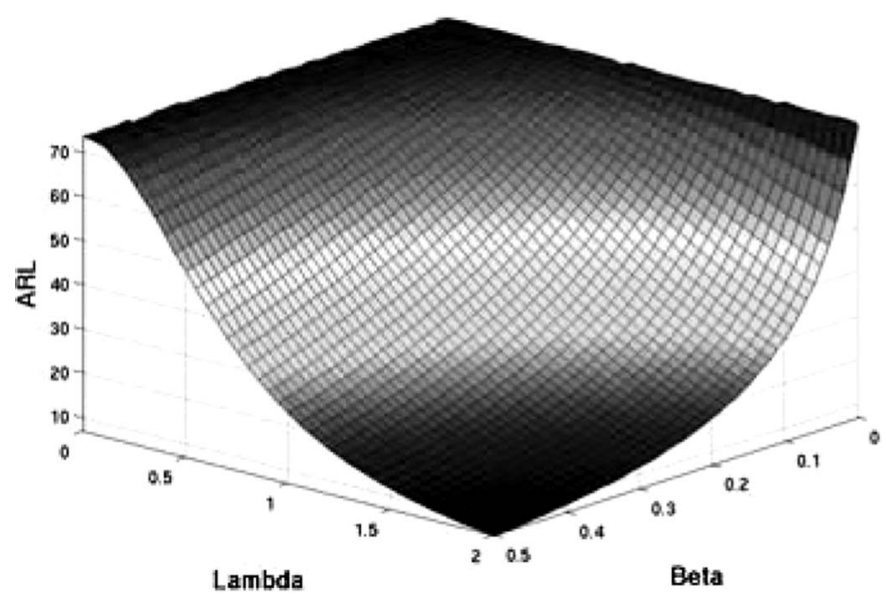

Figure 4. Evolution of the global ARL obtained with the eight rules combined in the case of a non symmetrical mixture. 


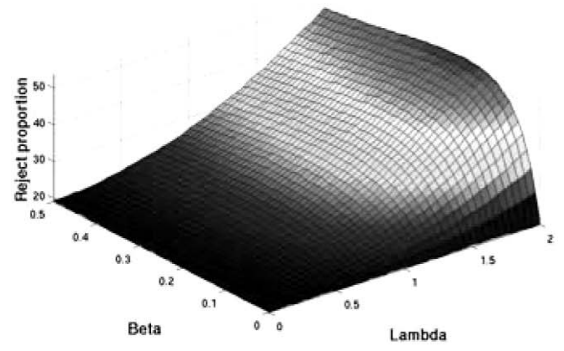

(a)

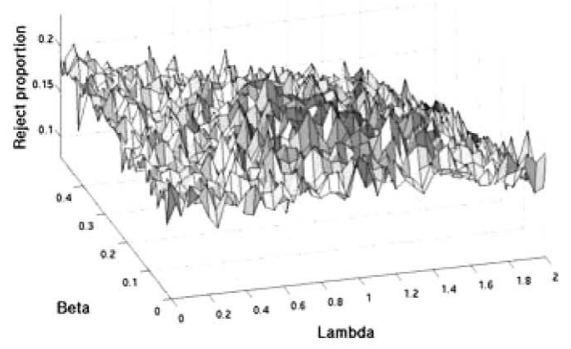

(b)

Figure 5. Percentage of detection of the mixture due to (a) Rule 1 and (b) Rule 8 (noise is due to the very low probability of detection of Rule 8 , and could be reduced by running many more simulations).

These results show that, for these two simple types of mixture that Rule 8 is designed to detect, the abnormality is almost always detected sooner by another rule; Rules 1 and 5 being globally the most sensitive to mixture patterns. Therefore, Rule 8 can be considered as simply useless in its present definition.

\section{Modification of the Mixture Detection Rule}

As far as the authors know, no theoretical or practical justification exists in literature for the design of Rule 8 and the previous results show that it is in fact useless in practical mixture cases. Therefore, the following modification of Rule 8 is proposed in order to obtain a mixture detection rule with an individual sensitivity comparable to that of the seven other rules and also more able to detect a mixture when it is effectively present.

Rule 8 (modified): Five points in a row on both sides of centreline with none in Zone $C$.

\subsection{In-Control Process: Power of the Modified Rule 8}

The number of points in sequence was chosen to obtain an individual FAR of this modified mixture detection rule approximately equal to $0.34 \%$ : close to that of the seven other rules. The impact of such a modification of Rule 8 is all the more visible when a combination of the eight rules is studied. Table 5 presents the values of the global ARL for the same three control charts used in Table 2, but with the modified Rule 8 . Contrary to Table 2, the modified Rule 8 leads to a significant decrease in the ARL.

\section{Table 5}

ARL Values for three combinations of rules with the modified Rule 8 (the values into brackets correspond to the Western definition of Rule 2 that considers only eight following points)

\begin{tabular}{lccc}
\hline Combination & $\mathrm{C}_{123456}$ & $\mathrm{C}_{1234567}$ & $\mathrm{C}_{12345678 \text { (modified) }}$ \\
\hline $\mathrm{ARL}$ & $79.0(70.0)$ & $73.5(65.8)$ & $68.3(61.8)$ \\
\hline
\end{tabular}


Table 6

Influence of the modified Rule 8 on the percentage of false alarms obtained by each rule for two control charts for the Nelson definition of Rule 2 (the values into brackets correspond to the Western definition)

\begin{tabular}{lcccccccc}
\hline Combination & Rule 1 & Rule 2 & Rule 3 & Rule 4 & Rule 5 & Rule 6 & Rule 7 & Rule 8 \\
\hline 1234567 & 19.5 & 11.9 & 15.9 & 10.7 & 11.1 & 22.0 & 8.9 & \\
& $(17.5)$ & $(22.0)$ & $(13.9)$ & $(9.3)$ & $(11.7)$ & $(19.4)$ & $(6.2)$ & \\
12345678 & 17.4 & 10.6 & 14.2 & 9.4 & 11.6 & 18.1 & 6.2 & 12.5 \\
& $(5.8)$ & $(19.8)$ & $(12.7)$ & $(8.3)$ & $(10.5)$ & $(16.2)$ & $(5.5)$ & $(11.1)$ \\
\hline
\end{tabular}

Additionally, Table 6 where the percentage of detection of each abnormal pattern is given, shows that the pattern of the mixture rule is detected approximately in $12.5 \%$ of the cases. So the modified Rule 8 significantly contributes to the global power of the control chart.

\subsection{Symmetrically Mixed Process: Power of the Modified Rule 8}

When the simulated data is symmetrically mixed, the global ARL obtained with the modified Rule 8 combined with the seven others is smaller than that obtained with the usual Rule 8 . This difference is more pronounced for weakly mixed data. Indeed, as shown in Fig. 6, the influence of the modification of Rule 8 is less and less visible when $\lambda$ increases $(\lambda \geq 0.5)$. This shows that even with the modified Rule 8 , a strong mixture is detected earlier by the other rules when $\lambda$ increases. This result is confirmed by the percentage of detection of each abnormal pattern plotted in Fig. 7. Indeed, for $\lambda \leq 1.5$, the modified Rule 8 is infringed in up to $20 \%$ of the samples, however when $\lambda$ increases further, Rules 1 and 5 are still

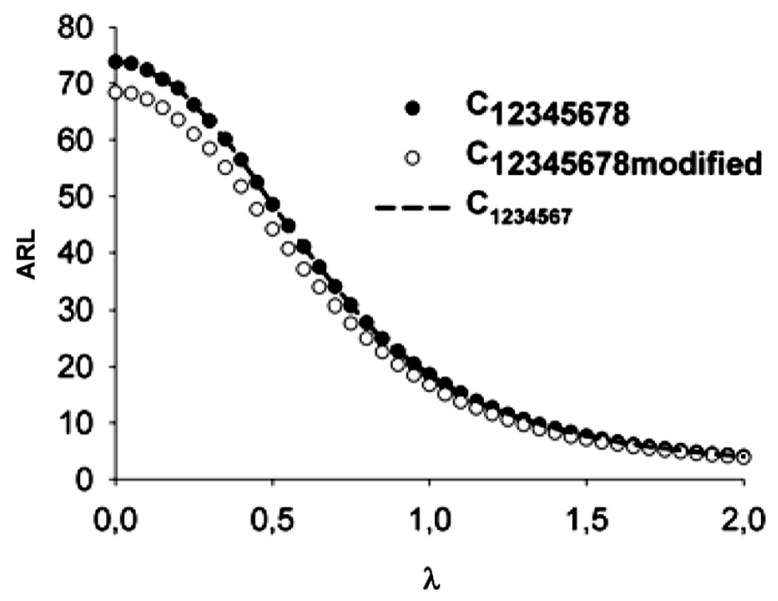

Figure 6. Comparison of the ARL obtained in the case of control charts combining the eight rules with either the usual Rule 8 or the modified one. 


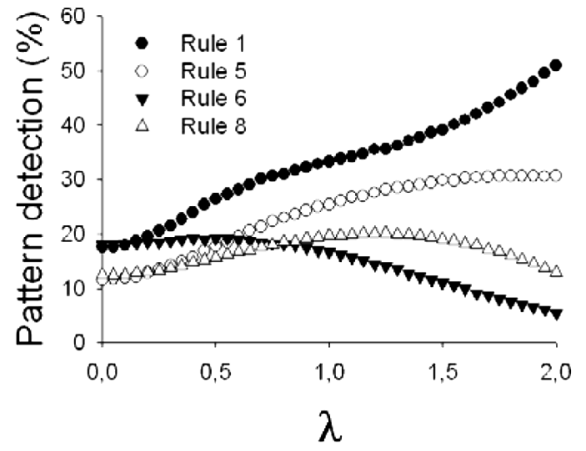

(a)

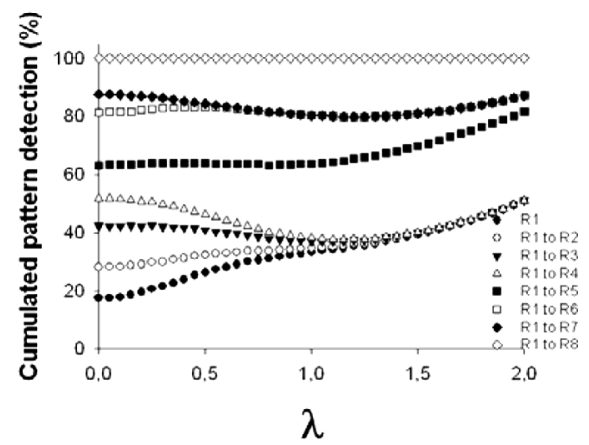

(b)

Figure 7. Percentage of detection of the mixture due to (a) Rules 1, 5, 6 and to the modified Rule 8. The cumulated percentages of detection shown in (b) highlight the fact that the modified Rule 8 plays a major role in the detection of a symmetrical mixture.

the most frequently infringed. This means that the modified Rule 8 is a powerful tool to detect the presence of rather weakly mixed data, that other rules are not always able to detect quickly. Additionally, for strongly mixed data the modified Rule 8 still detects a mixture in more than $15 \%$ of the simulations which is the actual cause of the out-of-control situation. Consequently, even for strongly mixed data, the modified Rule 8 is interesting since it enables to identify the special cause responsible for the drift from normality. The curve of the percentage of detection of the modified Rule 8 shown in Fig. 7(a) presents two distinct regions. On the one hand, for $0 \leq \lambda \leq 1.2$, the modified Rule 8 detects more and more frequently the presence of a mixture as $\lambda$ increases. However, for $\lambda \geq 1.2$, the percentage of detection of Rule 8 decreases when $\lambda$ increases, whereas the percentage of detection of Rule 1 starts to grow exponentially. This is typical of a competition between two mechanisms. During the first stage $(0 \leq \lambda \leq 1.2)$ the probability of occurrence of the pattern of the modified Rule 8 and of Rule 1 increases in a similar manner and their respective ARLs decrease in the same way, whereas during the second stage $(\lambda \geq 1.2)$ the ARL of Rule 1 becomes much smaller than that of Rule 8 (mainly because it needs fewer points to react) and puts it in the background.

\subsection{Non-Symmetrically Mixed Process: Power of the Modified Rule 8}

In the case of non symmetrically mixed data, the global ARL evolution is very similar to that shown in Fig. 4 but the surface is shifted to lower values, which means that the modification of Rule 8 increases the global sensitivity of the $C_{12345678}$ control chart. The evolutions of the percentage of detection for Rules 1 and 8 are plotted in Fig. 8. They are very similar to that plotted in Fig. 5, but the surface of Rule 1 is lowered (like those of Rule 2-7, not shown), whereas the percentage of detection of the modified Rule 8 is strongly increased compared to that of the usual Rule 8 . For non symmetrical mixtures, the modified Rule 8 proves to be more effective for weakly mixed data (low $\lambda$ and $\beta$ ). For strongly mixed data Rule 1 (and also Rule 5, not shown) are more quickly infringed and the modified Rule 8 is less useful. Therefore, here again, for subtle mixtures, which the first seven rules 


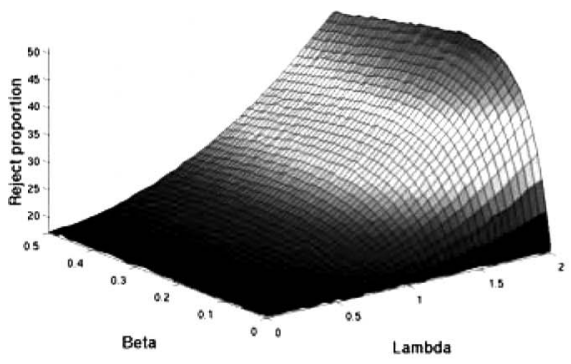

(a)

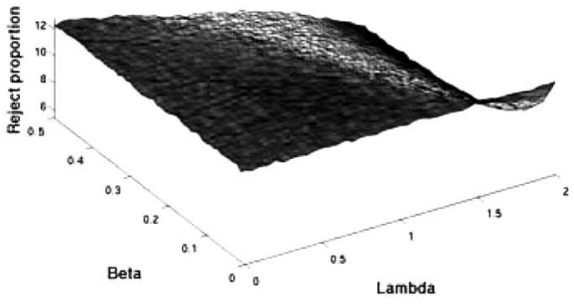

(b)

Figure 8. Percentage of detection of the mixture due to (a) Rule 1 and (b) the modified Rule 8.

are not very efficient to detect, the modified Rule 8 turns out to be powerful since it decreases the global ARL and enables to identify the presence of a mixture in more than $12 \%$ of the simulations. Nevertheless, when the mixture is more and more pronounced, the rules that need fewer points to react (Rules 1 and 5, for example) are infringed before Rule 8.

\section{Conclusion}

The FAR of the usual mixture detection rule used in SPC control charts was highlighted to be much lower (approximately 30 times) than the target Type-I error $(\approx 0.27 \%)$ used for the design of the seven other rules. This discrepancy leads to the fact both in-control and out-of-control data (presence of a mixture) Rule 8 has been found to be virtually useless. As no theoretical or practical justification has been found in literature a modification of the mixture detection rule is proposed to improve its sensitivity. Firstly, this modification leads to an individual Type-I error close to $0.34 \%$ which means that the eight rules can be considered as approximately equally sensitive. Secondly, this modification allows Rule 8 to increase the sensitivity of control charts when used in conjunction with any combination of the seven other rules. When a mixed population is simulated on purpose, the modified Rule 8 turns out to be much more efficient than the usual one. Additionally, it is found to be powerful in the detection of weakly mixed data, whereas for very pronounced mixtures Rules 1 and 5 remain the first ones to react, since they need fewer points. Therefore the modified Rule 8 turns out to be complementary to the seven other rules in the presence of a mixed population. It increases the global power of the $\mathrm{C}_{12345678}$ control chart (and of all the other combinations of rules) which detects sooner the out-of-control situation and enables to better identify the actual cause of defect in the presence of a mixture (either symmetrical or not).

\section{Acknowledgments}

We wish to thank Véronique Hague for her assistance with the English translation of this article. 


\section{References}

Adams, B. M., Lowry, C., Woodall, W. H. (2001). The use (and Misuse) of false alarm probabilities in control chart design. In: Lenz, H. J., Wetherill, Wilrich, P.-T. H., eds. Frontiers in Statistical Quality Control 6. Heidelberg: Physica Verlag.

Champ, C. W. (1992). Steady-state run length analysis of a Shewhart quality control chart with supplementary runs rules. Commun. Statist. Theor. Meth. 21(3):765-777.

Champ, C. W., Woodall, W. H. (1987). Exact results for Shewhart control charts with supplementary runs rules. Technometrics 29(4):393-399.

Charnes, J. M. (1995). Tests for special causes with multivariate autocorrelated data. Comput. Operat. Res. 22(4):443-453.

Efron, B., Tibshirani, R. J. (1993). An Introduction to the Bootstrap. New York: Chapman \& Hall.

Minnotte, M. C. (1992). A Test of Mode Existence with Applications to Multimodality. Ph.D. thesis, Rice University, Department of Statistics.

Mueller, D. W., Sawitzki, G. (1991). Excess mass estimates and tests for multimodality. JASA 86:738-746.

Nelson, L. S. (1984). The Shewhart control chart-tests for special causes. J. Qual. Technol. 16(4):237-239.

Nelson, L. S. (1985). Interpreting Shewhart X control charts. J. Qual. Technol. 17(2):114-116.

Shewhart, W. A. (1931). Economic Control of Quality of Manufactured Product. New York: Van Nostrand.

Walter, E., Philpot, J. W., Clement, J. (1991). False signal rates for the Shewhart control chart with supplementary runs tests. J. Qual. Technol. 23(3):247-252.

Western Electric Company (1956). Statistical Quality Control Handbook. Indianapolis, IN: AT\&T.

Zhang, S., Wu, Z. (2005). Designs of control charts with supplementary runs rules. Comput. Indust. Eng. 49:76-97. 